\title{
On Systems of Small Sets with No Large $\Delta$-Subsystems
}

\author{
A. V. KOSTOCHKA ${ }^{1} \dagger$, V. RÖDL $L^{2}$ and L. A. TALYSHEVA \\ ${ }^{1}$ Institute of Mathematics, Siberian Branch of the RAS, \\ Novosibirsk-90, 630090, Russia \\ (e-mail: sasha@math.nsc.ru) \\ 2 Department of Mathematics and Computer Science, Emory University, \\ Atlanta, GA 30322, USA \\ (e-mail: rodl@mathcs . emory . edu)
}

Received 9 September 1997; revised 29 May 1998

\begin{abstract}
A family of $k$ sets is called a $\Delta$-system if any two sets have the same intersection. Denote by $f(r, k)$ the least integer so that any $r$-uniform family of $f(r, k)$ sets contains a $\Delta$-system consisting of $k$ sets. We prove that, for every fixed $r, f(r, k)=k^{r}+o\left(k^{r}\right)$. Using a recent result of Molloy and Reed [5], a bound on the error term is provided for sufficiently large $k$.
\end{abstract}

\section{Introduction}

A family $\mathscr{F}$ of sets is called $r$-uniform if, for every $F \in \mathscr{F},|F|=r$ holds. A family of sets is called a $\Delta$-system if any two sets have the same intersection. Define $f(r, k)$ to be the least integer so that any $r$-uniform family of $f(r, k)$ sets contains a $\Delta$-system consisting of $k$ sets. Erdős and Rado [4] proved that

$$
(k-1)^{r}<f(r, k)<r !(k-1)^{r},
$$

and conjectured that, for each $k$, there exists a constant $C_{k}$ so that $f(r, k)<C_{k}^{r}$. Erdős (see [3]) offered $\$ 1000$ for a proof or disproof of this for $k=3$. In this note we consider the case when $r$ is fixed and $k$ is large enough.

Abbott, Hanson and Sauer [1] completely solved the case $r=2$, as follows.

$\dagger$ This work was partially supported by grant XRM1-181 of the Cooperative Grant Program of the Civilian Research and Development Foundation.

$\$$ This work was partially supported by grant 96-01-01614 of the Russian Foundation for Fundamental Research. 
Theorem A. (Abbott, Hanson and Sauer [1])

$$
f(2, k)= \begin{cases}k(k-1), & \text { if } k \text { is odd, } \\ (k-1)^{2}+(k-2) / 2, & \text { if } k \text { is even. }\end{cases}
$$

Abbott and Hanson [2] obtained an upper bound for $r=3$, as follows.

Theorem B. (Abbott and Hanson [2]) For $k \geqslant 7$,

$$
f(3, k) \leqslant 1.8 k(k-1)^{2}+8(k-1)^{2} .
$$

In this note, we apply the Pippenger-Spencer theorem [6] to show that, for fixed $r$, the lower bound in (1.1) is asymptotically (in $k$ ) tight.

Theorem 1. Let $r$ be fixed and $k$ be sufficiently large. Then

$$
f(r, k)=k^{r}+o\left(k^{r}\right) .
$$

Using a recent result of Molloy and Reed [5], we estimate the error term for sufficiently large $k$ as follows.

Theorem 2. Let $r$ be fixed and $k$ be sufficiently large. Then there exists a constant $c_{r}$ such that

$$
f(r, k) \leqslant k^{r}\left(1+c_{r} k^{-2^{-r}}\right) .
$$

\section{Background}

Let $H=(V, E)$ be a hypergraph with the vertex-set $V$ and the edge-set $E$. The degree $\operatorname{deg}_{H}(v)$ of a vertex $v \in V$ is the number of edges in $H$ containing $v$. Similarly, the codegree $\operatorname{codeg}(v, w)$ of a pair of vertices is the number of edges in $H$ containing both $v$ and $w$. Let $D(H)=\max \left\{\operatorname{deg}_{H}(v) \mid v \in V\right\}$ and $C(H)=\max \left\{\operatorname{codeg}_{H}(v, w) \mid v, w \in V\right\}$. If $C(H)=1$ then $H$ is called linear.

An edge-colouring of a hypergraph $H$ is proper if the edges of the same colour are vertex-disjoint, that is, form a matching.

Theorem C. (Pippenger and Spencer [6]) Let $r$ be fixed and $D$ be sufficiently large. Then, for each $r$-uniform hypergraph $H$ with $D(H) \leqslant D$ and $C(H)=o(D)$, there exists a proper edge-colouring with $D+o(D)$ colours.

Unfortunately, the theorem does not say which $D$ is 'sufficiently large' for a given $r$ and how small are $o(D)$. The situation with $o(D)$ for $C(H)=1$ was improved recently by Molloy and Reed, as follows.

Theorem D. (Molloy and Reed [5]) Let s be fixed and D be sufficiently large. Then there exists $c_{s}$ such that, for each s-uniform linear hypergraph $H$ with $D(H) \leqslant D$, there exists a proper edge-colouring with $D\left(1+c_{s}(\log D)^{6} D^{-1 / s}\right)$ colours. 
We shall also use the following folklore observation.

Observation E. Let $\mathscr{F}$ be an r-uniform family not containing a $\Delta$-system of size $k$, and let $W$ be a subset of the set $\bigcup_{F \in \mathscr{F}} F$. Further, let $\mathscr{F}_{W}=\{F \backslash W \mid F \in \mathscr{F}, W \subset F\}$. Then $\mathscr{F}_{W}$ is an $(r-|W|)$-uniform family not containing a $\Delta$-system of size $k$. In particular,

$$
|\{F \in \mathscr{F} \mid W \subset F\}|=\left|\mathscr{F}_{W}\right|<f(r-|W|, k) .
$$

\section{Proofs}

Proof of Theorem 1. First we prove Theorem 1 by induction on $r$. For $r=1$, it is trivial. For $r=2$, it follows from Theorem A. Suppose that Theorem 1 is proved for all $r<r_{0}$. Let $\mathscr{F}$ be an $r_{0}$-uniform family not containing a $\Delta$-system consisting of $k$ sets, where $k$ is sufficiently large. Let $H=(V, E)$ be the hypergraph with $E=\mathscr{F}$ and $v$ be an arbitrary vertex in $H$. By Observation $\mathrm{E}$ and then by the induction assumption, we have

$$
\left|\mathscr{F}_{\{0\}}\right|<f\left(r_{0}-1, k\right)=k^{r_{0}-1}+o\left(k^{r_{0}-1}\right) .
$$

In other words, $D(H) \leqslant k^{r_{0}-1}+o\left(k^{r_{0}-1}\right)$. Similarly, $C(H) \leqslant k^{r_{0}-2}+o\left(k^{r_{0}-2}\right)$. Thus, for some $D=k^{r_{0}-1}+o\left(k^{r_{0}-1}\right)$, we have $D(H) \leqslant D$ and $C(H)=o(D)$. By Theorem $\mathrm{C}$, the edges of $H$ can be partitioned into $t=D+o(D)=k^{r_{0}-1}+o\left(k^{r_{0}-1}\right)$ matchings $\mathscr{M}_{1}, \mathscr{M}_{2}, \ldots, \mathscr{M}_{t}$. Any matching is a $\Delta$-system. Thus, $\left|\mathscr{M}_{j}\right|<k$ for each $j$. It follows that $|\mathscr{F}|=|E|<k t=k^{r_{0}}+o\left(k^{r_{0}}\right)$.

To prove Theorem 2, we need the following fact.

Lemma 3. Let $H_{i}=\left(V_{i}, E_{i}\right)$ be an r-uniform hypergraph such that

(a) the family $E_{i}$ does not contain a $\Delta$-system of size $k$,

(b) $\left|e \cap e^{\prime}\right| \leqslant r-i$ for each $e, e^{\prime} \in E_{i}$.

Then $E_{i}$ can be partitioned into $k\left(1+c_{\left(\begin{array}{c}r \\ i\end{array}\right)}(\log k)^{6} D^{1-1 /\left(\begin{array}{l}r \\ i\end{array}\right)}\right)$ subsets $E_{i+1, j}$ such that $\left|e \cap e^{\prime}\right| \leqslant$ $r-i-1$ for each $e, e^{\prime} \in E_{i+1, j}$.

Proof. Consider the auxiliary hypergraph $G_{i}$ whose vertices are $(r-i)$-subsets of $V_{i}$, and $\left(\begin{array}{c}r \\ r-i\end{array}\right)$ vertices of $G_{i}$ form an edge if and only if the union of the corresponding $(r-i)$-subsets of $V_{i}$ is an edge in $H_{i}$. Thus there is one-to-one correspondence between edge sets of $H_{i}$ and $G_{i}$.

First observe that $G_{i}$ is simple. Indeed, if two distinct vertices of $G_{i}$ belong to at least two common edges, then the two corresponding distinct $(r-i)$-subsets of $V_{i}$ are contained in two common edges of $H_{i}$, a contradiction to (b).

Also, due to (b), the set of edges in $H_{i}$ containing a given $(r-i)$-subset of $V_{i}$ is a $\Delta$-system. Thus, $D\left(G_{i}\right)<k$, and applying Theorem D to $G_{i}$, we partition $E\left(G_{i}\right)$ into $k\left(1+c_{\left(\begin{array}{c}r \\ i\end{array}\right)}(\log k)^{6} k^{-1 /\left(\begin{array}{l}r \\ i\end{array}\right)}\right)$ matchings. Note that the corresponding partition of $E_{i}$ satisfies the statement of the lemma. 
Proof of Theorem 2. We are ready to prove Theorem 2. Let $\mathscr{F}$ be an $r$-uniform family not containing a $\Delta$-system consisting of $k$ sets, and let $H=(V, E)$ be the hypergraph with $E=\mathscr{F}$. Clearly, $H_{1}=H$ satisfies the conditions of Lemma 3 for $i=1$. Applying the lemma, we get that every hypergraph $H_{2, j}=\left(V\left(H_{2, j}\right), E_{2, j}\right)$ satisfies the conditions of Lemma 3 for $i=2$ and so on. Note that, after $r-1$ steps, 'matchings' in $H_{r-, j}$ correspond to real matchings in $H_{1}=H$. Thus, we have split $E$ in at most

$$
t=k^{r-1} \prod_{i=1}^{r-1}\left(1+c_{\left(\begin{array}{c}
r \\
i
\end{array}\right)}(\log k)^{6} k^{-1 /\left(\begin{array}{l}
r \\
i
\end{array}\right)}\right)
$$

matchings. Let $c_{r}^{\prime}=\max \left\{c_{(r)} \mid 1 \leqslant i \leqslant r-1\right\}$. Then

$$
\begin{aligned}
t & \leqslant k^{r-1} \prod_{i=1}^{r-1} \exp \left\{c_{r}^{\prime}(\log k)^{6} k^{-1 /\left(\begin{array}{c}
r \\
i
\end{array}\right)}\right\} \\
& \leqslant k^{r-1} \exp \left\{r c_{r}^{\prime}(\log k)^{6} k^{-1 /\left({ }_{[r / 2}^{r}\right)}\right\} .
\end{aligned}
$$

For large $k$, the last expression is at most $k^{r-1}\left(1+k^{-2^{-r}}\right)$. As above, no matching can contain $k$ or more edges. Thus, $|\mathscr{F}|<k \cdot k^{r-1}\left(1+k^{-2^{-r}}\right)$, as required.

\section{A remark}

M. Molloy and B. Reed have informed the authors that they are able to prove that the error term in the Pippenger-Spencer theorem (Theorem C) is at most

$$
c_{r}(\log D)^{\text {const }} D\left(\frac{C(H)}{D}\right)^{1 / r} \text {. }
$$

In the proof of Theorem 1 , we have $C(H) / D \leqslant 1 /(k+o(k))$. Then, repeating the proof of Theorem 1, we get the bound $f(r, k) \leqslant k^{r}\left(1+k^{(-1+\epsilon) / r}\right)$.

\section{References}

[1] Abbott, H. L., Hanson, D. and Sauer, N. (1972) Intersection theorems for systems of sets. $J$. Combin. Theory Ser. A 12 381-389.

[2] Abbott, H. L. and Hanson, D. (1974) On finite $\Delta$-systems. Discrete Math. 8 1-12.

[3] Erdős, P. (1975) Problems and results on finite and infinite combinatorial analysis. In Infinite and Finite Sets (Colloq. Keszthely 1973), Vol. I, Colloq. Math. Soc. J. Bolyai, 10, North Holland, Amsterdam, pp. 403-424.

[4] Erdős, P. and Rado, R. (1960) Intersection theorems for systems of sets. J. London Math. Soc. 35 85-90.

[5] Molloy, M. and Reed, B. (1997) Asymptotically better list colourings. Preprint.

[6] Pippenger, N. and Spencer, J. H. (1989) Asymptotic behavior of the chromatic index for hypergraphs. J. Combin. Theory Ser. A $5124-42$. 